\title{
Exploring the Relationship between the Use of Learning Technologies and Student Success in the Engineering Classroom
}

\section{Mr. Robert Matthew DeMonbrun, University of Michigan}

Matt DeMonbrun is a Ph.D. Candidate at the Center for the Study of Higher and Postsecondary Education (CSHPE) in the School of Education at the University of Michigan. His research interests include college student development theory, intergroup interactions, and teaching and learning practices and how they relate to student learning outcomes in engineering education.

\section{Mr. Michael Geoffrey Brown, University of Michigan}

Michael Brown is a doctoral candidate in the Center for the Study of Higher and Post-Secondary Education at the University of Michigan. 


\section{Exploring the Relationship between the Use of Learning Technologies and Student Success in the Engineering Classroom}

Prior research on the relationship between learning analytics data and student performance indicates that a large portion of students who are classified as academically struggling never recover from these failures [1]. Information about why these students never recover is lacking, however, because many analyses only focus on end of course grades to investigate student failure [2]. These models fail to account for the types of behaviors that might occur during the semester, which contribute to these failures, or what behaviors might encourage student success after initial setbacks in the course. By focusing on weekly behaviors, researchers, instructors, and course designers alike can examine the smaller events that occur from week-to-week, which may predict successful or risky behaviors in the course. This research paper presents findings from a study on the impact of Learning Management Systems (LMSs) on student success in the classroom. Because of the amount of data available from the LMS, we can examine student performance on a week-to-week basis using data available from using digital course technologies. This contrasts with the use of end of course final grades, which do not provide researchers with the type of nuanced behaviors that might lead to success or failure throughout the duration of the semester.

\section{Use of Instructional Tools and Academic Recovery}

Very little research exists on the types of factors that may predict academic success or failure within a course, particularly through the use of online instructional tools and learning management systems. Waddington et al. [3] found that few digital course technologies significantly impacted positive academic performance. In fact, only exam preparation resources positively affected academic performance in courses. Students who utilized their online exam preparation tool were predicted to have much better chance of passing the course, when compared to students who stuck with only current methods of preparing for exams in the course (e.g., note-taking, recitation of lecture notes). However, studies like the one above, only take a single snapshot of student performance (at the end of the semester) and use binary metrics like pass/fail to determine success or failure in the course [4].

In this study, we investigate the role of time and the use of online instructional tools to uncover the relationship between the use of the tools at various times during the semester and student success or failure in the course. Using an existing Early Warning System (EWS) called "Student Explorer" (see below) that classified students into three risk categories, we use event history methods on students' performance data from the EWS and their interaction with the E2Coach system to answer the following research questions:

RQ1) (a) Which instructional technologies, if any, help to predict the likelihood of students entering explore or engage classifications? (b) Which instructional technologies, if any, help to predict the likelihood of students exiting explore or engage classifications? 
RQ2) Does the timing of students' use of these technologies precede, coincide with, or lag their experience of academic difficulty? In other words, do students use these tools throughout the duration of the semester, or only when they experience academic difficulty?

\section{Methodology}

We employed weekly academic classifications in an early warning system (EWS) for students in an undergraduate engineering course at a research-intensive university in the Midwest. Coupled with the EWS, we used data from students' use of various instructional technologies during the course through a digital coaching application called E2Coach. The E2Coach system provides students with a variety of resources including: weekly help messages, exam preparation (before the exam) and reflection (after the exam) tools, a weekly checklist of tasks that will help students prepare for the class, a grade calculator so students can estimate their grade based on past and planned future performance, and various online systems for reviewing academic material. These methods are discussed in detail below.

\section{Sample}

Students in our sample were enrolled in an introductory programming course in the Electrical Engineering and Computer Science program. This course is a prerequisite for many computer science and computer engineering students, while also serving a substantial non-major population at the institution. The course has been redesigned using the E2Coach system to encourage students to use strategies that promote academic success in the course and correct risky academic behaviors early in the semester. Given that this is a " 100 level" academic course, students often take this course in their first or second semester at the institution, and thus, the course also serves as a platform for academic success strategies throughout their college experience.

Our sample includes 695 students who took this course in the Winter 2016 academic semester. The course involved lectures twice a week and a weekly lab section. All instructors used the same instructional resources, including all assignments and exams. Demographics for the course are listed in Table 1. Students in the sample were $61 \%$ male and predominantly White $(49.6 \%)$ or Asian (31.3\%).

\section{Variables}

The EWS gives a weekly categorization of each student's performance for each course and designation of performance as a status of "Encourage" (green - student performing at or above the course mean), "Explore" (yellow - students performing below the course mean), or "Engage" (red - students in the lowest quartile of performance), based on various metrics, including: currently available grade data, students' interaction with course tools and materials, and students' performances when compared to their peers in the course. 
Table 1

Demographic Characteristics of Participants $(n=695)$

\begin{tabular}{lrr}
\hline Characteristics & $\mathrm{N}$ & $\%$ \\
\hline Female & 249 & 38.72 \\
White & 319 & 49.61 \\
Black & 17 & 2.64 \\
Hispanic & 27 & 4.2 \\
Asian & 201 & 31.26 \\
Other & 79 & 12.29 \\
& & \\
High School GPA & Mean & $\mathrm{SD}$ \\
ACT & 3.82 & 0.16 \\
Math Placement & 30.83 & 2.82 \\
Prior College GPA & 20.08 & 4.15 \\
\hline
\end{tabular}

The dependent variable for our analysis is a dichotomous variable measuring the change in level for each student's weekly classification ( $1=$ change in classification; $0=$ no change in classification). For example, all students begin in the "encourage" (green) classification. If a student changed from "encourage" (green) to "explore" (yellow) in the third week of the semester, he or she is flagged as entering the "explore" classification for that week (i.e., the dependent variable would be flagged as " 1 " instead of " 0 "). Students changing from "encourage" (green) or "explore" (yellow) to "engage" (red) would be flagged as entering the "engage" classification.

We also created dependent variables for exiting out of either the "explore" or "engage" classifications. Exiting the "explore" classification represents a status change from "explore" (yellow) to "encourage" (green), and exiting the "engage" classification represents a status change from "engage" (red) to either "explore" (yellow) or "encourage" (green). Students must have entered the classification in order to exit it, and once students enter the classification, the exit models indicate how long it takes before the student exits the classification. For example, if a student entered into the "explore" classification in the third week of the semester and exited the "explore" classification in the fifth week of the semester, the dependent variable in week 2 (the second week in the classification) would be flagged as " 1 " indicating that they also exited the classification in that week.

In addition to the demographic characteristics listed in Table 1, we also drew log data from online records of students' use of various instructional technologies utilized in the course. Students are provided access to a digital coaching system, called E2Coach. The E2Coach system provides a variety of resources for this course, as discussed below:

Exam Preparation: A tool providing resources and strategies for preparing for exams. The exam preparation tool asks students to identify what resources they will use to prepare for exam. In the model, this is measured as the number "click throughs" for each page in the exam preparation guide. 
Exam Reflection: A tool providing resources and strategies for reflecting on exams. The exam reflection tool provides students the opportunity to review and reflect upon how they prepared for an exam. In the model, this is measured as the number "click throughs" for each page in the exam reflection guide.

Grade Calculator: An interactive tool that students can use to estimate their grade based on current and future performance. In the model, Grade Calculator is a binary indicator for whether or not the student used the Grade Calculator in a given week.

As part of the course, students are also asked to use two online systems for reviewing and submitting the second assignment in the course. This assignment is heavily weighted (points) and is generally the first assignment in the course where students experience academic difficulty.

Assignment 2 - First Submission: Once the assignment is released to the students (generally in the third week of class), students have an option to submit a revised version of the assignment each weekday and are provided feedback from the instructional team regarding errors in the assignment, or ways to achieve a better grade. In the model, the first submission variable represents whether or not their first draft submission of this assignment was in a given week.

Assignment 2 - Last Submission: Once students feel comfortable with their effort on the assignment and the potential grade given, they may submit a final version for review. Students may submit this final version during any week after the assignment is released to students. The assignment is due in the seventh week of the semester, and although students may submit the assignment late, points are deduced from each day late. The last submission variable represents whether or not their final draft submission of this assignment was in a given week.

\section{Analysis}

Our analysis utilizes event history (or hazard) modeling to determine the probability that students will either enter or exit the "explore" or "engage" classification in a given week. Considering classifications in the Student Explorer system are reported on a weekly basis, we utilized a discrete-time hazard model for this analysis, as our data is reported in discrete-time periods. In other words, while we do not know the exact date and time that the student entered the classification (this is dependent upon when the instructional team grades their assignments), we are given a classification update each week in discrete time periods.

Discrete-time hazard models employ binary responses $\left(\mathrm{y}_{\mathrm{ti}}\right)$, where the outcome represents whether the event occurred $(1=y e s ; 0=$ no) during sequential time periods $(t)$ for each individual (i). We created a weekly observation for whether an individual student entered or exited a classification ("explore" or "engage"). The probability $\left(\mathrm{p}_{\mathrm{ti}}\right)$ is then estimated for each individual (i) to experience the event during each time interval ( $t$ ), given that no event has occurred prior to the start of $\mathrm{t}$ : 


$$
\mathrm{p}_{\mathrm{ti}}=\operatorname{Pr}\left(\mathrm{y}_{\mathrm{ti}}=1 \mid \mathrm{y}_{\mathrm{t}-1}, \mathrm{i}=0\right)
$$

$\mathrm{p}_{\mathrm{ti}}$ is known the discrete-time hazard function because it represents the probability of the individual entering or exiting a classification during a specific weekly interval. After determining the probabilities for each individual's time hazard, the data is fit to a binary response model (i.e., logistic regression model):

$$
\log \left(\mathrm{p}_{\mathrm{ti}} / 1-\mathrm{p}_{\mathrm{ti}}\right)=\alpha \mathrm{D}_{\mathrm{ti}}+\beta \mathrm{x}_{\mathrm{ti}}
$$

In this model, $p_{t i}$ represents the probability of the event occurring for the individual (i) during the time interval $(t), D_{t i}$ is a vector of functions representing the total cumulative hazard during the duration by interval (t) with coefficients $(\alpha)$, and $\mathrm{x}_{\mathrm{ti}}$ is a vector of covariates with coefficients $(\beta)$. Each individual receives a baseline hazard function (represented by $\mathrm{D}_{\mathrm{ti}}$ ), while the covariates can either increase or decrease the hazard function for each individual. The results of the logistic regression model presented below are provided in terms of odds ratios for ease of discussion. In total, four models were estimated for our analysis: entering the "explore" classification, entering the "engage" classification, exiting the "explore" classification, and exiting the "engage" classification. We did not include multiple events in our model. In other words, if a student entered a classification, exited it promptly, and then reentered it later in the semester, we did not record this reentry in our analysis; however, this occurred in less than 3\% of students in our sample.

\section{Results}

Before beginning our analysis on students' use of instructional technology in the course, we fitted a baseline model using only time as a predictor to examine its impact on entry into or exit out of EWS classifications. In doing so, we are able to model and examine the "riskiest" points of the academic term (i.e., when most students enter either "explore" (yellow) or "engage" (red) classifications. The baseline model for entry into the EWS is illustrated in Figure 1. Figure 1 depicts the odds of entering each classification (designated by either the yellow "explore" or the red "engage" lines) for each week in the semester. For example, week 8 appears to be the riskiest time for students to enter the "explore" classification of the model, with their odds being about 70 times more likely to enter this classification when compared to the other weeks in the model. Both weeks 3 and 8 were the riskiest weeks for entry into "engage" classification of the model, with odds being between 15 and 20 times more likely to enter when compared to other weeks. 


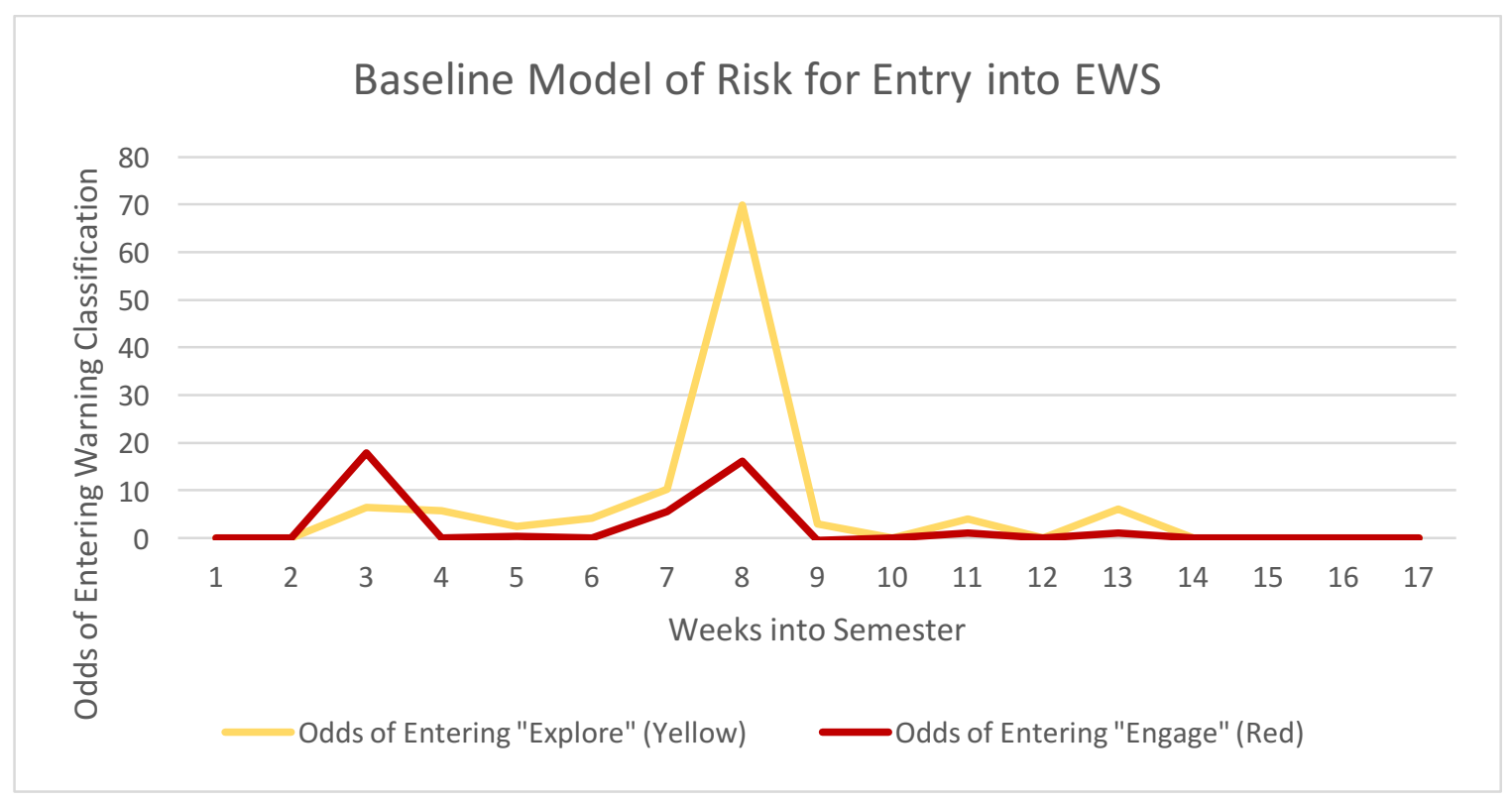

Figure 1. Baseline Model of Risk for Entry into EWS.

Figure 2 depicts a similar analysis for exit out of the model for each of the classifications. This analysis examines approximately how long, after entering the classification, it takes students to exit the classification by calculating the odds of exiting the model each week after entry. In this figure, the $\mathrm{x}$-axis represents the number of weeks after entering the classification, while the y-axis represents the increased odds of exiting the model in that week. For example, in the "explore" (yellow) model, students have the greatest odds of exiting this classification in weeks 1 and 3. Students are 14 times more likely to exit the model in week 1 and 18 times more likely to exit the model in week 3, when compared to other weeks in the analysis. For the "engage" (red) model, the only significant week to exit the model is during week 2 when students are two times more likely to exit the classification. More discussion on the explanations for this analysis are provided in the discussion section.

\section{Predictors for Entry Models}

After depicting the baseline model, we entered several demographic characteristics, academic measures, and instructional technology predictors into the hazard model to examine the overall impact of these controls on entry into and exit out of each classification. The results of this analysis are presented in Table 2 . All coefficients are presented as odds ratios (i.e., the odds of entering/exiting the classification) for ease in interpretation.

As indicated in Table 2, no demographic characteristics are significant predictors for entering either the "explore" or "engage" classifications of the EWS. Across the academic measures, increases in students' ACT scores and college GPA (prior to the current academic term) lower their odds of entering each model. For example, as college GPA increases by one grade point, the odds of entering into the "explore" model decrease by a little over half (54\%). These results are even greater for the "engage" model, where the same GPA increase results $73 \%$ lower odds of entering into this classification. 
Students' ACT score is also a significant predictor and perhaps more powerful than college GPA as each one point increase in ACT score decreases the odds of entering the "explore" classification by $14 \%$.

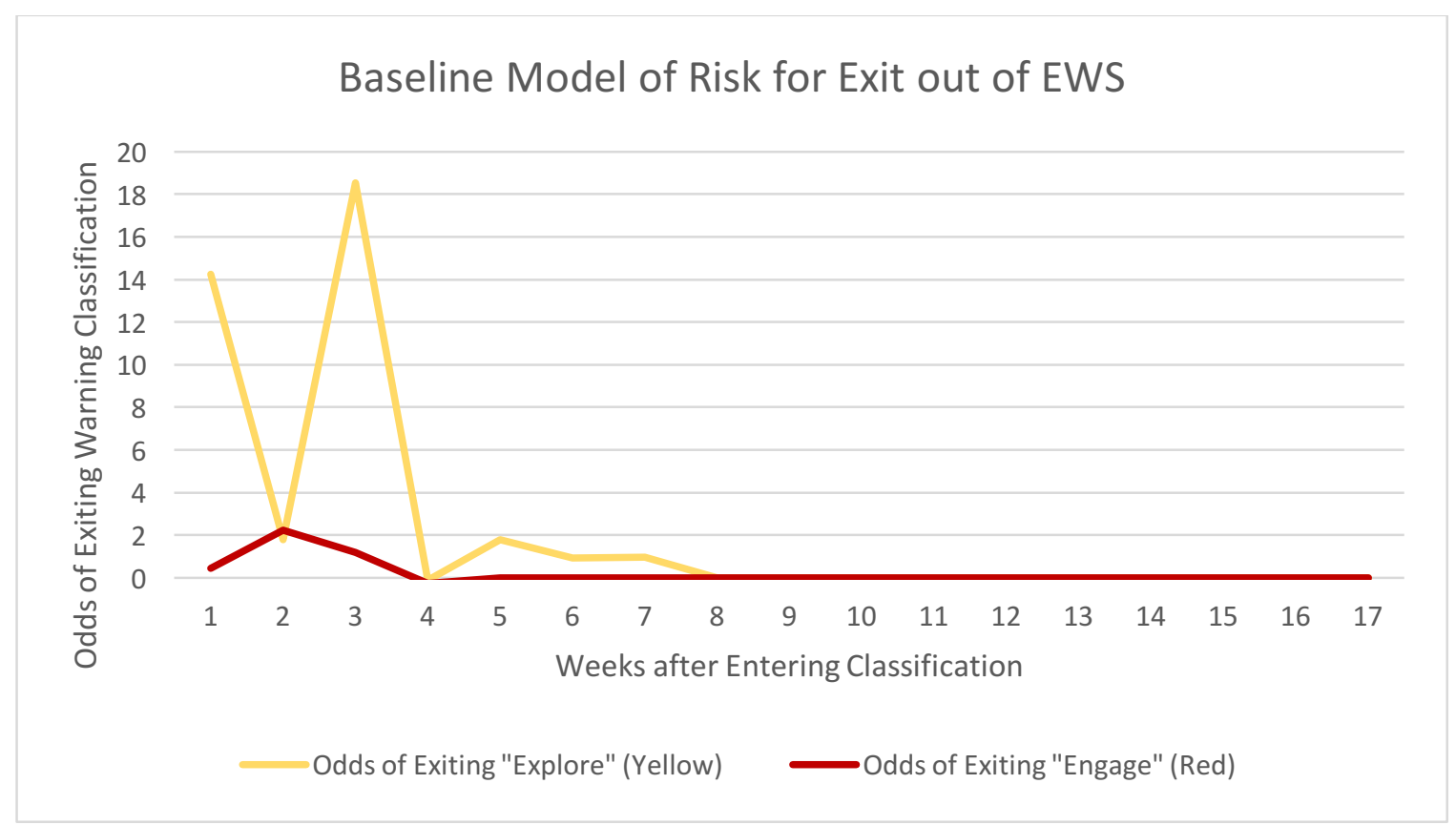

Figure 2. Baseline Model of Risk for Exit out of EWS.

Across the instructional technology tools, each additional "click through" of a page in the exam preparation tool resulted in a $2 \%$ decrease in the odds of entering the "explore" classification $(\mathrm{p}<0.05)$. Although this appears to be a small decrease in odds, the average number of "click throughs" for this course was 38.2, which when compounded, could result in significant changes. The only two other significant predictors in this model were the first and last submission dates for the second assignment. For example, for each additional week that students did not submit a final draft of the homework, resulted in a 56\% greater probability of entering into the "explore" classification. This could be as a result of either late grade penalties for submitting after the deadline, reduction in the score from poor quality as a result of starting the assignment late, or some combination of both. Additionally, each additional week that students did not submit their first draft of the assignment resulted in being over twice as likely to enter the "engage" classification. Again, this could be as a result of grade penalties or poor preparation of the assignment.

While the model in Table 2 indicates what predictors, overall, impact a students' potential entry into either the "explore" or "engage" classifications, they do not account for the variation in these predictors across time. In Table 3, we present the interaction between weeks into the semester and each of the instructional technology tools. For brevity in interpretation, we only present those tools which significantly impacted entry into these classifications, although all predictors were included in the full model. 
Table 2

Odds Ratios of Predictors for Entering and Exiting EWS Classifications

\begin{tabular}{|c|c|c|c|c|}
\hline & \multicolumn{2}{|c|}{ Entry } & \multicolumn{2}{|c|}{ Exit } \\
\hline & Yellow & Red & Yellow & Red \\
\hline \multicolumn{5}{|l|}{ Gender (vs. Male) } \\
\hline Female & 1.17 & 1.16 & 0.90 & 0.66 \\
\hline \multicolumn{5}{|l|}{ Race (vs. White) } \\
\hline Black & 0.19 & 0.86 & 1.00 & 1.00 \\
\hline Hispanic & 0.76 & 1.00 & 5.94 & 1.00 \\
\hline Asian & 1.28 & 0.57 & 0.53 & 10.49 \\
\hline Other & 1.11 & 0.36 & 5.33 & 0.79 \\
\hline \multicolumn{5}{|l|}{ Academic Characteristics } \\
\hline HS GPA & 1.00 & 1.55 & 5.14 & 0.23 \\
\hline $\mathrm{ACT}$ & $0.86^{*}$ & 1.06 & 1.16 & 0.72 \\
\hline Math Placement & 1.00 & 0.96 & 1.12 & 0.91 \\
\hline College GPA & $0.46^{* *}$ & $0.27 * *$ & $3.55^{*}$ & 0.63 \\
\hline \# of Units Taken & 0.93 & 1.09 & 1.00 & 2.06 \\
\hline \multicolumn{5}{|l|}{ Instructional Tools } \\
\hline Exam Prep & $0.98 *$ & 1.00 & 1.03 & 1.02 \\
\hline Grade Calculator & 1.00 & 1.00 & 1.03 & 0.97 \\
\hline Exam Reflection & 1.00 & 0.99 & 1.01 & 1.06 \\
\hline Assn \#2 - First Submission & 0.94 & $2.10^{*}$ & 1.19 & 0.31 \\
\hline Assn \#2 - Last Submission & $1.56^{*}$ & 0.77 & 0.70 & 4.34 \\
\hline
\end{tabular}

${ }^{*} \mathrm{p}<0.05 ; * * \mathrm{p}<0.01 ; * * * \mathrm{p}<0.001$

As indicated in Table 3, week 6 was a significant predictor for students' first submission of assignment 2 . In other words, students who waited until the sixth week of the course to submit their first draft of the assignment (this assignment was opened to students in week 3 ) were nearly 9 times more likely to enter into the "explore" classification when compared to other weeks. Students who waited until week 7 to submit the first draft were nearly three and a half times more likely to enter the "engage" classification, and one and a half times more likely to do so if they waited until week 8 . These hazard rates are additive in that students who first submitted in week 8 were exposed to the risks in week 7 and week 8.

Students' final submission date was also a significant predictor of entry into the "explore" classification. Students who waited until week 7 to make their final submission had 1.7 times greater odds of entering this classification, while students who waited until week 8 had an additional one and a half times greater odds of doing so. Alternatively, use 
of the exam prep tool decreased students" odds of entering into both the "explore" and "engage" classifications. Students who began using this tool in week 3 were $3 \%$ less likely to enter the "engage" classification. Furthermore, students who used the tool in week 7 were $6 \%$ less likely to enter the "explore" classification, and $4 \%$ less likely in week 8 to enter the "engage" classification.

Table 3

Impact of Use of Instructional Tools on Weekly Risk of Entering EWS Classification

\begin{tabular}{|c|c|c|c|c|c|c|c|c|c|c|c|c|}
\hline & \multicolumn{2}{|c|}{ Week 3} & \multicolumn{2}{|c|}{ Week 4} & \multicolumn{2}{|c|}{ Week 5} & \multicolumn{2}{|c|}{ Week 6} & \multicolumn{2}{|c|}{ Week 7} & \multicolumn{2}{|c|}{ Week 8} \\
\hline & Y & $\mathrm{R}$ & Y & $\mathrm{R}$ & Y & $\mathrm{R}$ & Y & $\mathrm{R}$ & Y & $\mathrm{R}$ & Y & $\mathrm{R}$ \\
\hline $\begin{array}{l}\text { Assn2 - First } \\
\text { Submission }\end{array}$ & 1.58 & 1.06 & 1.00 & 1.71 & 3.07 & 1.25 & $8.94 *$ & N/A & 0.73 & $3.46^{*}$ & 1.00 & $1.51^{*}$ \\
\hline $\begin{array}{l}\text { Assn2 - Last } \\
\text { Submission }\end{array}$ & 0.69 & 1.17 & 1.29 & 0.54 & 0.58 & 1.13 & 0.15 & 0.00 & $1.70 *$ & 0.48 & $1.30 *$ & 1.21 \\
\hline Exam Prep & 1.00 & $0.97 * *$ & 0.99 & 0.92 & 1.02 & 0.96 & 0.97 & 1.01 & $0.94 *$ & 0.98 & 0.99 & $0.96^{* *}$ \\
\hline
\end{tabular}

Initial findings indicate that students who use the tools starting at the beginning of the term have decreased odds of experiencing academic difficulty during the term, particularly for those who use the exam preparation guides from the E2Coach system. Additionally, students who utilize checklist items, especially in regards to early submission of course projects, are half as likely to enter the "Explore" or "Engage" classifications. For those students who experience academic difficulty in the "Explore" classification, the use of the exam reflection tools doubles their odds of recovering in the course (i.e., their classification shifts from "Explore" to "Encourage"). The full paper will expand upon the tools used in the E2Coach platform and the rest of the analyses from our full statistical model.

\section{Predictors for Exit Models}

Table 2 also presents the demographic characteristics, academic measures, and instructional technology predictors that impact students' likelihood of exiting each of the EWS classifications. We only found one significant predictor for exiting either the "explore" or "engage" model. For every one point increase prior college GPA, students had three and a half times greater odds of exiting the "explore" classification. No significant indicators were found for exiting the "engage" classification. Table 4 presents the interaction between weeks in either the "explore" or "engage" classification and the each of the instructional technology tools and its impact on exiting these respective classifications. Again, for brevity in interpretation, we only present those tools which significantly impacted entry into these classification, although all predictors were included in the full model. 
Table 4

Impact of Use of Instructional Tools on Weekly Risk of Exiting EWS Classification

\begin{tabular}{|c|c|c|c|c|c|c|c|c|c|c|c|c|}
\hline & \multicolumn{2}{|c|}{ Week 1} & \multicolumn{2}{|c|}{ Week 2} & \multicolumn{2}{|c|}{ Week 3} & \multicolumn{2}{|c|}{ Week 4} & \multicolumn{2}{|c|}{ Week 5} & \multicolumn{2}{|c|}{ Week 6} \\
\hline & $\mathrm{Y}$ & $\mathrm{R}$ & $\mathrm{Y}$ & $\mathrm{R}$ & Y & $\mathrm{R}$ & Y & $\mathrm{R}$ & Y & $\mathrm{R}$ & $\mathrm{Y}$ & $\mathrm{R}$ \\
\hline $\begin{array}{l}\text { Exam } \\
\text { Reflection } \\
\text { Grade }\end{array}$ & 1.89 & 1.65 & 1.49 & 2.16 & $5.04 *$ & 0.88 & 1.00 & 0.34 & 1.36 & 1.24 & 0.57 & 1.00 \\
\hline Calculator & 0.60 & 0.87 & 3.16 & 0.93 & 1.59 & $4.81 *$ & 1.00 & $9.21 *$ & N/A & $10.82 *$ & N/A & 1.00 \\
\hline
\end{tabular}

As indicated in Table 4, the exam reflection tool was a significant predictor for exiting the "explore" model after three weeks. After three weeks in the "explore" classification, students who used the exam reflection tool had five times greater odds of exiting that classification. The grade calculator had similar effects for the "engage" model. After three weeks in the "engage" classification, students who used the grade calculator had 4.8 times greater odds of exiting the classification, an additional 9.2 times greater odds of exiting in week 4 , and an additional 10.8 times greater odds of exiting in week 5 . The interpretations for each of these findings is presented below.

\section{Discussion and Implications}

There are several points of discussion after reviewing our findings for each of the hazard models. First, as indicated in our baseline model of risk, week 8 encompassed the greatest risk for entering either of our classifications. This is likely because of the amount of points at risk around this point in the semester (during midterms), as both the second assignment and first exam were implemented around this time period. This finding, however, is meaningful because it illustrates where students are most likely to falter in the course, and thus, additional attention can be applied by course instructors, teaching assistants, and academic advisors/coaches in supporting students through this period. From the baseline models of exiting each of the classifications, we can see that after the fourth week in the "explore" and "engage" models, students' probability of exiting either of these classifications drops significantly. This draws two different conclusions. First, students who exit either of these classifications do so in significant numbers between the second and fourth weeks. Second, those who remain in the classification after four weeks are significantly less likely to exit afterwards. This provides significant feedback for instructors to encourage bringing grades up as soon as possible, rather than relying on a final exam to pull students out of trouble.

Additionally, while some academic characteristics were significant indicators in predicting entry into or exit out of each of the classifications, the instructional tools were also extremely valuable in mitigating the risk of entering the model, or helping students after they entered the model to exit quickly. Although information on the first and last submission was not a tool, per se, it does provide meaningful feedback to instructors and academic advisors/coaches in helping motivate students to push forward on this assignment. A unique aspect about the E2Coach system is the ability to tailor messages to students who meet certain criteria. Therefore, if students have not submitted the second 
assignment by the fifth week, a message could be sent informing them that submitting after that week might subject them to moderate risk in performing poorly in the course. It should be noted that the assignment was due after the seventh week into the semester, so the risk indicated in the "engage" classification for the first submission in weeks seven and eight were likely as a result of either starting the assignment just days before the deadline, or submitting the assignment late.

Among the instructional tools, use of the exam preparation, exam reflection, and grade calculator tools were significant in either helping students stay out of the EWS classifications, or making a successful exit out of the system soon thereafter. The exam preparation tool appeared to assist students in thinking ahead about the first exam, where a large percentage of overall course points were at risk, thus allowing them to perform better on the exam and stay out of the "explore" and "engage" classifications. For those students who did enter these classifications, the exam reflection and grade calculator tools helped to pull them out of trouble. The exam reflection tool appears to have given students an opportunity to reflect on what went wrong, particularly with the second assignment and first exam, and move forward with a better plan for success. The grade calculator could have also helped students better understand what grades they needed to make on subsequent assignments/exams to pull themselves out of these classifications. Overall, these findings demonstrate that these instructional tools are helpful in keeping students out of academic trouble in the course, or assisting them out quickly afterwards, and future messaging to students from the E2Coach system should reflect the impact that these tools have on student success.

\section{Limitations}

As with any study, there are several limitations to our findings. First, this study was a cross-sectional examination of one course over one academic semester. We did not conduct analyses to determine whether the number of pass/fail students or the grade distribution was representative of other courses in this major field, nor if this was common across each semester. Second, although the odds of entering and exiting each of these classifications appears alarming, it should be noted that, out of the 695 students in the course, only 130 students entered the "explore" classification and 109 students entered the "engage" classification. (The "engage" classification did also include students who could have moved from "explore" to "engage" during the semester, so these students might have been double counted.) Of those who entered the "explore" classification, only 67 exited the model, while 64 exited the "engage" classification. Third, our models only included students' first time entering and exiting each model. Thus, our model is not predictive of whether or not students reentered either classification after exiting the first time. Finally, although the hazard models indicate which instructional tools were the most effective, they do not answer the question of why. We have speculated in the results section above as to how students might have used each of these tools (by their intended design), but this is not to say that this is exactly how each tool was used. Future analyses will incorporate qualitative data to discuss with students using each tool how it impacted their success in the course. 


\section{Future Directions}

Our analysis provides several interesting findings that we plan to follow-up with for future analyses. First, several of the instructional tools offered through the E2Coach system had a meaningful impact on students' probability of staying out of academic trouble, or quickly exiting either the "explore" or "engage" classifications. Additional research should attempt to investigate the underlying how and why these tools assist in this way. Second, although our analyses attempted to control for student demographics that might impact entry into or exit out of the EWS, we did not capture students' individual motivation to perform well in the course. We also did not control for academic major in our models. Students majoring in computer science or computer engineering might have more incentive to perform better, as opposed to non-majors in the course. Finally, wide-scale implementation of these analyses is needed to explore whether these results are consistent across semester and type of course. Different instructional tools may be more useful given a different student population, and perhaps, those students in upper-division courses who have persisted into their third and fourth years would be less impacted by a system like E2Coach and would require a different analysis to investigate academic difficulties and recovery.

\section{References}

1. M. G. Brown, R. M. DeMonbrun, S. Lonn, S. J. Aguilar, and S. D. Teasley, "What and when: the role of course type and timing in students' academic performance," in Proceedings of the Sixth International Conference on Learning Analytics \& Knowledge (pp. 459-468), Edinburgh, Scotland, 2016.

2. B. J. Zimmerman, "Investigating self-regulation and motivation: Historical background, methodological developments, and future prospects," American Educational Research Journal, vol. 45, no. 1, 2008.

3. R. J. Waddington, S. J. Nam, S. Lonn, and S. D. Teasley, "Practice (Exams) Make Perfect: Incorporating course resource use into an early warning system," Journal of Learning Analytics, in press.

4. L. R. Halverson, C. R. Graham, K. J. Spring, J. S. Drysdale, and C. R. Henrie, “A thematic analysis of the most highly cited scholarship in the first decade of blended learning research," The Internet and Higher Education, vol. 20, 2014. 